HJOG 2018, 17 (1), 9-12

\title{
Successful outcome birth in full term pregnancy velamentous insertion of the umbilical cord - vasa previa
}

\author{
Thanasas K. Ioannis, Papavasileiou Sofia \\ Department of Obstetrics - Gynecology of General Hospital in Trikala, Trikala, Greece
}

Correspondence

Ioannis K. Thanasas, MD, MSc, Efkli 33, 42100 Trikala, Greece, Tel.: 2431029103, 6944766469, E - mail: thanasasg@hotmail.com

\begin{abstract}
Disclosure of incident on our successful outcome childbirth in term pregnancy velamentous insertion of the umbilical cord - vasa previa. Pregnant firstborn with free individuals and obstetrical history covering the 39th week of pregnancy is introduced in Maternity Clinic of our with symptomatology of automatic start birth. Sudden and to thoroughly grade vaginal hemorrhage found immediately after technical rupture of fetal membranes accompanied by decelerations of the fetal heartbeat raised the possible diagnosis of velamentous insertion of the umbilical cord - vasa previa and the interest undergone emergency cesarean section. After removal of the fetus and placenta was there synovial adherence cord. The caesarean section was performed smoothly with physiological loss blood. There was no need for blood transfusion in neonates, as well as it did not take neonatal support. Following a smooth postoperative course left together with the postpartum at fifth day of hospitalization. At this paper on the basis of current data a brief literature review of rare disease entities this respect mainly the diagnosis, treatment and prognosis.
\end{abstract}

Key words: velamentous insertion of the umbilical cord - vasa previa, diagnosis, management, prognosis

\section{Introduction}

The synovial adhesion cord less frequently and often regional or copings adhesion among the most dangerous anatomical abnormalities of the umbilical cord and holds a special place in the challenge fetal distress from disorders of blood circulation in the umbilical vessels. As defined synovial adhesion is when the umbilical cord is not overgrown exclusively in chorionic network may, but at least in part, on fetal films. The umbilical vessels are separated on their way onto the film before reaching the periphery of the placenta. Embryonic vasculature associated with synovial adhesion cord and displayed in front of the viewing fate of fetal called precursor vessels ${ }^{1}$.

The velamentous insertion of the umbilical cord 
- vasa previa is very rare, but also threatening situation for the life of the newborn. The synovial adhesion cord estimated to be approximately $1 \%$ of monodymon pregnancies ${ }^{2}$, while in twins is approximately 7 - 8 times more often. Multiple gestations the incidence is estimated to be even greater, since it is more likely the umbilical blood vessels located between fetal membranes that separate the fetus ${ }^{3}$. Generally, the incidence of vascular precursor related to the synovial adhesion cord estimated that affects about one case per 2000 to 3000 childbirth $^{4}$.

In this article after the presentation of the event you attempt to breach the systematic arrangement and treatment of modern literature of a quick review of velamentous insertion of the umbilical cord - vasa previa, in particular in the diagnosis, treatment and prognosis, of which may help to reduce the increased risk of perinatal morbidity and mortality.

\section{Case report}

The description of case is on a pregnant woman on pregnant women firstborn with free individual and obstetrics story that traveled the 39th week of pregnancy, attended outpatient of our clinic with symptoms of automatic opening childbirth. Following the introduction of the interest in the delivery room, on clinical examination the cervical dilatation was assessed 3-4 centimeters. The embryo follicle was unbreakable and viewing the embryo was cephalic. The placenta, as assessed by ultrasonography was rear high position. The cardiotocography was satisfactory. Entered perfusion (Ringer $1000 \mathrm{ml}$ with 10 units oxytocin and delivery rate $40 \mathrm{ml}$ per hour) to strengthen the travails of the uterus and the progress of labor. The sudden and to thoroughly rhythm of vaginal hemorrhage found after the technical rupture of fetal hymen accompanied by deceleration of fetal heart rate raised the possible diagnosis of velamentous insertion of the umbilical cord - vasa previa and the interest undergone in a emergency cesarean section. After the removal of the fetus and placenta was found the synovial adherence cord - precursor vessels (Figure 1). The caesarean section was performed smoothly with normal blood loss. There was no necessary of execution blood transfusion in neonates and as the introduction in a Neonatology unit. After smooth postoperative course the postpartum together with the newborn left our clinic the fifth day of hospitalization with instruction for review at the outpatient clinic after the puerperium.

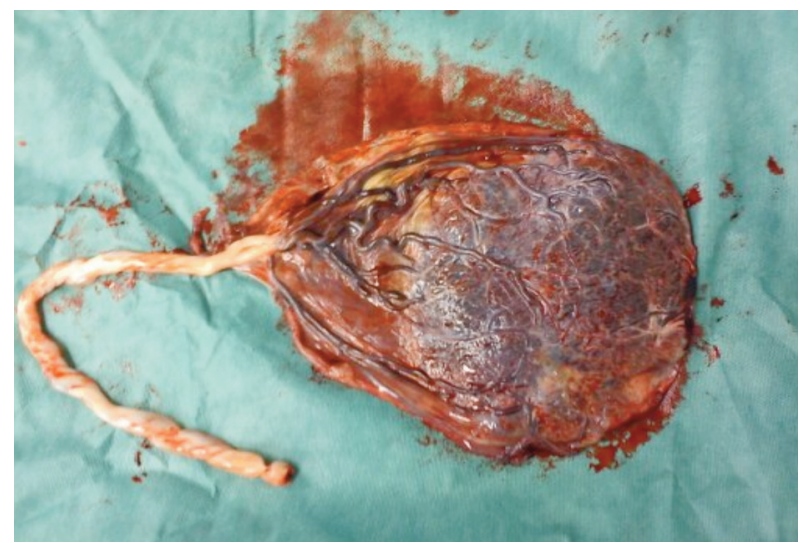

Figure 1. Velamentous insertion of the umbilical cord (our case). It is evident outgrowth cord from the embryo follicle and the removal of umbilical vascular embryonic membranes.

\section{Discussion}

Although formerly believed that synovial adhesion cord does not pose a problem to proper course of pregnancy, based on the newest literature data most of the researchers believe that the situation increased the rates releases premature birth, low birth weight neonates, fetal difficult fetal $\operatorname{loss}^{5}$ as well as the rates cesarean section ${ }^{6}$. The clinical diagnosis of synovial adherence and precursor vessel during the pregnancy and prior to dilation of the cervix or before the rupture of fetal membranes is impossible. The clinically suspected diagnosis of precursor vessels is possible to put the onset of 
labor in those cases that during the vaginal examination fumble vases in membranes covering the projected fate of the baby through the fully or partially dilated cervix. The sudden and significant degree of vaginal bleeding that is detected after the rupture of fetal membranes and accompanied from fetal tachycardia, corrugated fetus rate or by other signs fetal distress (our case) may indicate embryonic bleeding after rupture precursor vessel. In the event has not occurred rupture of fetal membranes, the diagnosis of precursor vascular can be substantiated by amnion overview ${ }^{7}$.

Prenatal diagnosis of velamentous insertion of the umbilical cord - vasa previa is not easy to put. The diagnostic value of ultrasound has sensitivity $67 \%$ and specificity of $100 \%$ in the second quarter of pregnancy ${ }^{8}$, while in first quarter diagnosis is also likely ${ }^{9}$. The best time for prenatal diagnosis is the second trimester of pregnancy ${ }^{10}$. Despite that however, the widespread use of three - dimensional ultrasonography and the transvaginal Power Doppler sonography has increased significantly the diagnostic accuracy of these situation before childbirth ${ }^{11}$. Also, Magnetic - resonance tomography will be useful in the diagnosis of precursor vessels in each case that the relationship between the position of the placenta and the position of suspects under consideration can not be clearly appreciated by ultrasound ${ }^{12}$.

The therapeutic approach of pregnant with velamentous insertion of the umbilical cord - vasa previa depends on the time of diagnosis. If the diagnosis is made during the pregnancy, usually in the second quarter, the planned caesarean will be done as far as possible towards the end of pregnancy and after administration of corticosteroids between the 28th and 32nd week of pregnancy with purpose pulmonary maturation of embryos and is a suitable way labor completion. Emergency caesarean section has a place in all cases in which the diagnosis is made during the duration of labor after rupture of the fetal membranes, as in our case. If the fetus born alive, may require the execution of blood transfusion because of the major fetal anemia ${ }^{13}$.

The prognosis for the fetus depends on early diagnosis and the rate of loss of fetal blood. Although it has been reported in the literature that fetal mortality can reach up to $50 \%-75 \%$ of cases after the rupture precursor vessel during the childbirth, early prenatal diagnosis based on modern imaging techniques has significantly reduced the index of perinatal morbidity and mortality ${ }^{14}$.

\section{Conclusion}

The velamentous insertion of the umbilical cord - vasa previa affected is not frequent. The modern diagnostic approach of these rare disease entities is a very important step for the proper course of pregnancy and the success of childbirth. The early recognition of the symptoms and the risk factors related to the disease as well as the correct application of modern advanced technology now permit early diagnosis and prompt proper handling to avoid a sudden neonatal death.

\section{References}

1. Elsaves KM, Trout AT, Friedkin AM, Liu PS, Bude RO et al. Imaging of the placenta: a multimodality pictorial review. Radiographics 2009; 29: 1371 - 1391.

2. Supelveda W, Rojas I, Robert JA, Schnapp C, Alcalde JL. Prenatal detection of velamentous of the umbilical cord: a prospective color Doppler ultrasound study. Ultrasound Obstet Gynecol 2003; 21: $564-569$.

3. Raithatha N, Patel M, Patel R, Chauhan N. Velamentous insertion of umbilical cord in twin pregnancy. NJIRM 2011; 2: 121 - 123.

4. Marie Helen Beall. Velamentous insertion and vasa previa. Updated: Jan 13, $2012 \mathrm{http}: / /$ www.emedicine.medscape.com/article/26247 
0-oreview

5. Ralsanen S, Georgiadis L, Harju M, Keski Nisula L, Heinonen S. Risk factors and adverse pregnancy outcomes among births affected by velamentous umbilical cord insertion: a population - based register study. Eur J Gynecol Reprod Biol 2012; 165: 231 - 234.

6. Rao KP, Belogolovkin V, Yankowitz J, Spinnato JA 2nd. Abnormal placentation: evidence based diagnosis and management of placenta previa, placenta accrete, and vasa previa. $\mathrm{Ob}$ stet Gynnecol Surv 2012; 67: 503 - 519.

7. Clark JFJ. Fetal heart monitoring in detecting velamentous insertion of the umbilical cord. J Natl Med Assoc 1984; 76: 292 - 294.

8. Hasegawa J, Matsuoka R, Ichizuka K, Sekizawa A, Okai T. Velamentous cord insertion: significance of prenatal detection to predict perinatal complications. Taiwan J Obstet Gynecol 2006; 45: $21-25$.

9. Sepulveda W. Velamentous insertion of the umbilical cord: a first - trimester sonographic screening study. J Ultrasound Med 2006; 25: $963-968$.

10. Kanda E, Matsuda Y, Kamitomo M, Maeda T, Mihara K, Hatae M. Prenatal diagnosis and management of vasa previa: a 6 - year review. J Obstet Gynaecol Res 2011; 37: 1391 - 1396.
11. Markov D, Ivanov S, Markov P, Diavolov V, Nikolov A et al. Velamentous insertion of the umbilical cord - diagnosis and management. Akush Ginecol (Sofia) 2009; 48: 3 - 10.

12. Kikuchi A, Uemura R, Serikawa T, Takakuwa K, Tanaka K. Clinical significances of magnetic resonance imaging in prenatal diagnosis of vasa previa in a woman with bilobed placentas. J Obstet Gynaecol Res 2011; 37: 75 - 78.

13. Gagnon R, Morin L, Bly S, Butt K, Cargill YM et al. Guidelines for the management of vasa previa. J Obstet Gynaecol Can 2009; 31: 748 - 760.

14. Babovic I, Plecas D, Plecinac S, Antonovic 0. Vasa previa and postpartum hysterectomy in maternal Rh alloimmunization. Clin Obstet Gynecol 2011; 38: 421 - 423. 